C. TON-THAT et al.

\title{
Blue shift in the luminescence spectra of MEH-PPV films containing ZnO
}

\section{nanoparticles}

Cuong Ton-That ${ }^{*}$ and Matthew R. Phillips

Department of Physics and Advanced Materials, University of Technology Sydney, P.O. Box 123, Broadway, NSW 2007, Australia

Thien-Phap Nguyen

Institut des Matériaux Jean Rouxel, University of Nantes, 44322 Nantes Cedes 03, France

* Corresponding author. fax: +61.2.95142219, tel: +61.2.95142201.

Email address: cuong.ton-that@uts.edu.au

PACS: 72.80.Tm; 78.30.Jw; 78.67.Bf

Keywords: Conjugated polymer; Photoluminescence; PPV; Nanoparticles; ZnO 
C. TON-THAT et al.

\begin{abstract}
Luminescence properties of nanocomposites consisting of $\mathrm{ZnO}$ nanoparticles in a conjugated polymer, MEH-PPV, were investigated. Photoluminescence measurements reveal a blue shift in the emission spectrum of MEH-PPV upon incorporation of $\mathrm{ZnO}$ nanoparticles into the polymer film while the emission is increasingly quenched with increasing $\mathrm{ZnO}$ concentration. In contrast, the structure of the polymer and its conjugation length are not affected by the presence of $\mathrm{ZnO}$ nanoparticles (up to 16 weight \% $\mathrm{ZnO}$ ) as revealed by Raman spectroscopy. The blue shift and photoluminescence quenching are explained by the separation of photogenerated electron-hole pairs at the MEH-PPV/ZnO interface and the changing of the nanoparticles.
\end{abstract}

\title{
1. Introduction
}

Incorporating inorganic nanoparticles into conjugated polymer matrices is an area of current interest in the fields of optoelectronics and solar energy. This approach can take advantages of the beneficial properties of both materials: superior optoelectronic properties of conjugated polymers and high electron mobility of inorganic semiconductors [1]. In addition, polymers are technologically advantageous owing to the ease and flexibility of processing devices in solutions. There have been reports on polymer-inorganic hybrid solar cells using CdSe [2], $\mathrm{TiO}_{2}[3]$ and $\mathrm{ZnO}$ nanoparticles and nanopods [4, 5]. Experimental results have demonstrated that these hybrid devices provide the potential for improving the performance with the powerconversion efficiency better than those achieved with polymer-only devices. In this context, dispersing nanoparticles in a polymer matrix is of interest for both basic research and optoelectronic applications. 
C. TON-THAT et al.

In this paper we present an optical and chemical study of nanocomposites composed of $\mathrm{ZnO}$ nanoparticles and poly[2-methoxy-5-(2'-ethyl hexyloxy)-phenylene vinylene] (MEH-PPV). $\mathrm{ZnO}$ and MEH-PPV were chosen because these two materials are widely studied for their intriguing optoelectronic properties. In a type II heterojunction between a polymer and a wide band gap semiconductor such as the MEH-PPV/ZnO system, electrons can diffuse unimpeded from the polymer to the semiconductor. As the polymer is illuminated with photons of energy larger than the band gap, electron-hole pairs are generated. The electrons are injected into the conduction band of the semiconductor and can move along the nanoparticle network, ultimately being collected via an electrical contact in photovoltaic devices. The nature of the charge transfer process depends on the optical properties of two materials as well as the surface properties of the nanoparticles. The present study focuses on the luminescence properties of the MEH-PPV/ZnO nanocomposite and the energy transfer between the polymer and the nanoparticles.

\section{Experimental}

$\mathrm{ZnO}$ nanoparticles with an average diameter of $25 \mathrm{~nm}$ were chosen for this study to provide a large surface area-to-volume ratio without introducing quantum size effects (the Bohn radius of $\mathrm{ZnO}$ is $1.8 \mathrm{~nm}$.) The nanoparticles were obtained from Advanced Nanotechnology Ltd, Australia who fabricate undoped $\mathrm{ZnO}$ nanoparticles using a mechanochemical-processing ${ }^{\mathrm{TM}}$ technique. MEH-PPV (the molecular structure is shown in Fig 1) was prepared using an improved synthesis method described by Neef and Ferraris [6]. The polymer was dissolved in toluene to form solutions with a concentration of $8 \mathrm{mg} / \mathrm{ml}$. Composite samples were prepared by adding $\mathrm{ZnO}$ nanoparticles to MEH-PPV solutions, followed by ultrasonication for 1 hour to improve the dispersion of $\mathrm{ZnO}$ nanoparticles. Films are prepared by spin-coating onto glass or $\mathrm{KBr}$ substrates. Characterization of the nanocomposite films was performed by Raman 
C. TON-THAT et al.

spectroscopy using a Brucker RFS 100 spectrophotometer. Photoluminescence (PL) measurements were made using a Fluorolog-3 spectrophotometer with optical excitation at $480 \mathrm{~nm}$.

\section{Results and discussion}

The PL spectra of pure MEH-PPV and MEH-PPV/ZnO nanocomposites in the wavelength range from 500 to $800 \mathrm{~nm}$ are presented in Fig 2(a). In this range, MEH-PPV is primarily responsible for the luminescence emission with little contribution from $\mathrm{ZnO}$. The dominant peak of pure MEH-PPV at $607 \mathrm{~nm}$ is an emission characteristic of the PPV backbone that arises from the relaxation of excited $\pi$-electrons to the ground state, while the 635-nm peak is related to the interchain states $[7,8]$. The emission features are not significantly affected by the incorporation of nanoparticles; however, a remarkable blue-shift is observed for the composites. The wavelength shift depends on the $\mathrm{ZnO}$ content with a maximum shift of 11 nm observed for 2 weight \% (Figure 2(b)). Furthermore, incorporation of $\mathrm{ZnO}$ nanoparticles into MEH-PPV results in luminescence quenching of the polymer. Since the films are deposited on identical $\mathrm{KBr}$ substrates using the same deposition parameters, any variation in the PL emission is expected to result from the nature of the nanocomposites. At 16 weight \% $\mathrm{ZnO}, 60 \%$ of the original MEH-PPV emission intensity is lost. Considering the small amount of $\mathrm{ZnO}$ incorporated into the nanocomposite films, the luminescence quenching cannot be attributed to a reduction in the volume of the emissive material, but rather to either energy or exciton transfer from the polymer to ZnO. Although the Förster energy transfer mechanism has been recently invoked for PbS and CdSe nanoparticles incorporated in MEH-PPV [9, 10], it can be excluded in this case due to the lack of overlap between the $\mathrm{ZnO}$ absorption and MEH-PPV emission spectra. On the basis of the relative positions of the MEH-PPV and ZnO energy levels, the disassociation of excitions at the interface between the materials is 
C. TON-THAT et al.

energetically allowed (Figure 3). Exposure of the nanocomposite films to visible light ( $\lambda=$ $480 \mathrm{~nm}$ ) does not lead to the generation of electron-hole pairs in the bulk of $\mathrm{ZnO}$ since the photon energy is smaller than the band gap of the material, optical excitations occur entirely within the polymer matrix. The transfer of excitons produced by photo-excitation of MEHPPV to the $\mathrm{ZnO}$ conduction band should be responsible for the luminescence quenching (discussed further below). Similar behaviours have been reported for composite thin films of poly(3-hexylthiophene) and $\mathrm{ZnO}$ nanoparticles [4]. The polymer emission quenching in our case is more severe because of a larger surface area-to-volume ratio (smaller nanoparticles), leading to a higher possibility of charge transfer at the MEH-PPV/ZnO interface.

As a general trend, as more $\mathrm{ZnO}$ nanoparticles are incorporated into the film, the MEH-PPV luminescence quenching increases and a shift of the emission spectrum to shorter wavelengths is observed. However, the blue shift is less dramatic for the nanocomposite films with the ZnO content $>4$ weight \%. Even at the low nanoparticle contents used in the present study, the possible formation of nanoparticle aggregates may be envisaged, similar to our previous findings of $\mathrm{PPV} / \mathrm{SiO}_{2}$ composite films [11]. The reduced blue shift in the emission spectra for the nanocomposites with high $\mathrm{ZnO}$ concentrations is most likely due to an increase in the nanoparticle aggregation that would result in a lower number of interfaces with the nanoparticles.

A blue shift in the luminescence spectrum of a conjugated polymer is commonly associated with changes in the conjugation length. Upon incorporation of nanoparticles, bonds between a polymer chain and the nanoparticle surface may be formed, altering the electronic configuration of the polymer chain and hence the conjugation length [12]. However, this is not the case for the MEH-PPV/ZnO system. Figure 4 presents the Raman spectra of pure 
C. TON-THAT et al.

MEH-PPV and the composite films, which show no significant differences in either the shape or the intensity of the characteristic bands of MEH-PPV, suggesting no changes in the conjugation length [12]. For the composite films with the $\mathrm{ZnO}$ concentration $\geq 8$ weight $\%$, there is a weak band at $436 \mathrm{~cm}^{-1}$ attributable to the optical phonon $\mathrm{E}_{2}$ mode of $\mathrm{ZnO}$ [13]. All the spectra display Raman band frequencies at $1115 \mathrm{~cm}^{-1}$ (mixture of $\mathrm{C}-\mathrm{C}$ stretching and $\mathrm{C}-\mathrm{H}$ in-plane bending liberation), $1282 \mathrm{~cm}^{-1}$ (C=C stretching band of benzene), $1582 \mathrm{~cm}^{-1}$ (C-C stretching band of benzene) and $1624 \mathrm{~cm}^{-1}$ ( $\mathrm{C}=\mathrm{C}$ stretching band of vinyl group) [11, 14]. The triplet bands around $1582 \mathrm{~cm}^{-1}$ are particularly sensitive to the chain planarity and conjugation length of PPV-based polymers [15]. Careful inspection of the triplet indicates that the relative intensities of two bands at 1547 and $1624 \mathrm{~cm}^{-1}$ remain virtually unchanged for the nanocomposites with $\mathrm{ZnO}$ concentrations $\leq 16$ weight $\%$. These results indicate that the chemical structure and the gap energy of MEH-PPV are not affected by the incorporation of ZnO nanoparticles.

A plausible explanation for the blue shift of the luminescence spectrum is the effect of the electric field produced by excess electrons on the nanoparticle surface as suggested by Musikhin et al. [16]. The surface of $\mathrm{ZnO}$ nanoparticles is a strong perturbation of the lattice where there exists a high concentration of both shallow and deep defect levels $[17,18]$. To elucidate the surface chemical structure, we have conducted an X-ray photoelectron spectroscopy (XPS) analysis of the ZnO nanoparticles. The O/Zn atomic ratio, determined using the integrated intensities of the $\mathrm{O} 1 \mathrm{~s}$ and $\mathrm{Zn} 2 p_{3 / 2}$ levels, is $0.67 \pm 0.10$, which is significantly less than unity, indicating that the surface region of the nanoparticles is nonstoichiometric with a large number of oxygen vacancies. Furthermore, the oxygen vacancy $\mathrm{V}_{\mathrm{o}}^{+}$with one electron was found to be the most abundant oxygen-deficient-related defects in ZnO nanoparticles [19]. The energy level of the $\mathrm{V}_{\mathrm{o}}^{+}$centre is located at $\sim 1 \mathrm{eV}$ below the 
C. TON-THAT et al.

conduction band edge [20]. Each $V_{o}^{+}$centre can accept an electron to become a $V_{o}^{0}$ centre (oxygen vacancy occupied by two electrons). Upon the transfer of electrons from MEH-PPV, these electrons can populate the defect states, thus creating an electric field outside the nanoparticles. This will raise the energy of the lowest exciton state and lead to the blue shift of the luminescence. The absence of any changes in the Raman bands, together with the results from surface characterisation of the nanoparticles, indicates that, contrary to a previous report on the degradation of polymers [5], the blue shift is due to the charging of the $\mathrm{ZnO}$ nanoparticles. After sufficient illumination and all the defect centres are filled, trapped electrons can undergo non-radiative decays via surface states, or tunnel into the nanoparticle and relax radiatively or non-radiatively, depending on the nature of the final state. This mechanism also provides a route towards separation of electron-hole pairs which are photogenerated within MEH-PPV and explains the quenching of the polymer emission.

\section{Conclusions}

We have investigated the luminescence spectra of nanocomposites based on MEH-PPV with embedded ZnO nanoparticles. Results of PL measurements show a remarkable blue shift in the luminescence spectra upon addition of the $\mathrm{ZnO}$ nanoparticles, accompanied by substantial quenching of the emission. Raman spectroscopy indicates that the conjugation length and the chemical structure of the polymer are unaffected by the incorporation of the $\mathrm{ZnO}$ nanoparticles up to $16 \%$ by weight. The luminescence quenching is due to the transfer of photogenerated electrons from the polymer to $\mathrm{ZnO}$ while the blue shift is attributable to the electron charging of the nanoparticles.

\section{References}

[1] J. Nelson, Curr. Opin. Solid State Mat. Sci. 6, 87 (2002). 
C. TON-THAT et al.

[2] W. U. Huynh, J. J. Dittmer, and A. P. Alivisatos, Science 295, 2425 (2002).

[3] A. Petrella, M. Tamborra, M. L. Curri, P. Cosma, M. Striccoli, P. D. Cozzoli, and A. Agostiano, J. Phys. Chem. B 109, 1554 (2005).

[4] W. J. E. Beek, M. M. Wienk, and R. A. J. Janssen, Adv. Funct. Mater. 16, 1112 (2006).

[5] W. J. E. Beek, L. H. Slooff, M. M. Wienk, J. M. Kroon, and R. A. J. Janssen, Adv. Funct. Mater. 15, 1703 (2005).

[6] C. J. Neef and J. P. Ferraris, Macromolecules 33, 2311 (2000).

[7] R. Traiphol, N. Charoenthai, T. Srikhirin, T. Kerdeharoen, T. Osotchan, and T. Maturos, Polymer 48, 813 (2007).

[8] C. Ton-That, G. Stockton, M. R. Phillips, T. P. Nguyen, C. H. Huang, and A. Cojocaru, Polym. Int. 57, 496 (2008).

[9] T. W. F. Chang, S. Musikhin, L. Bakueva, L. Levina, M. A. Hines, P. W. Cyr, and E. H. Sargent, Appl. Phys. Lett. 84, 4295 (2004).

[10] L. Bakueva, S. Musikhin, M. A. Hines, T. W. F. Chang, M. Tzolov, G. D. Scholes, and E. H. Sargent, Appl. Phys. Lett. 82, 2895 (2003).

[11] S. H. Yang, T. P. Nguyen, P. Le Rendu, and C. S. Hsu, Thin Solid Films 471, 230 (2005).

[12] S. H. Yang, P. Le Rendu, T. P. Nguyen, and C. S. Hsu, Rev. Adv. Mater. Sci. 15, 144 (2007).

[13] X. Q. Wei, B. Y. Man, M. Liu, C. S. Xue, H. Z. Zhuang, and C. Yang, Physica B 388, 145 (2007).

[14] P. Damlin, C. Kvarnstrom, and A. Ivaska, Electrochim. Acta 44, 1919 (1999).

[15] Q. G. Zeng and Z. J. Ding, J. Phys.-Condes. Matter 16, 5171 (2004).

[16] S. Musikhin, L. Bakueva, E. H. Sargent, and A. Shik, J. Appl. Phys. 91, 6679 (2002). 
C. TON-THAT et al.

[17] A. van Dijken, E. A. Meulenkamp, D. Vanmaekelbergh, and A. Meijerink, J. Phys. Chem. B 104, 4355 (2000).

[18] C. Ton-That, M. R. Phillips, M. Foley, S. J. Moody, and A. P. J. Stampfl, Appl. Phys. Lett. 92, in press (2008).

[19] K. Vanheusden, W. L. Warren, C. H. Seager, D. R. Tallant, J. A. Voigt, and B. E. Gnade, J. Appl. Phys. 79, 7983 (1996).

[20] A. Janotti and C. G. Van de Walle, Phys. Rev. B 76, 165202 (2007). 
C. TON-THAT et al.

\section{Blue shift in the luminescence spectra of MEH-PPV films containing ZnO nanoparticles}

\section{Figure Captions}

Fig. 1. Molecular structure of MEH-PPV

Fig. 2. (a) PL spectra of pure MEH-PPV and MEH-PPV/ZnO nanocomposite films acquired at $300 \mathrm{~K}$. Films were deposited onto $\mathrm{KBr}$ substrates. Excitation was set at $\lambda=480 \mathrm{~nm}$. (b) Variations of the wavelength shift and the relative intensity of the PL spectrum as a function of $\mathrm{ZnO}$ weight fraction in the films.

Fig. 3. Energy level diagram for $\mathrm{ZnO}$ and $\mathrm{MEH}-\mathrm{PPV}(\mathrm{CB}=$ conduction band, $\mathrm{VB}=$ valence band, LUMO = lowest unoccupied molecular orbital, HOMO = highest occupied molecular orbital)

Fig. 4. Raman spectra of pure MEH-PPV and MEH-PPV/ZnO nanocomposites. There are no significant changes in the characteristic bands of MEH-PPV upon incorporation of the nanoparticles into the polymer. 
C. TON-THAT et al.

Figure 1

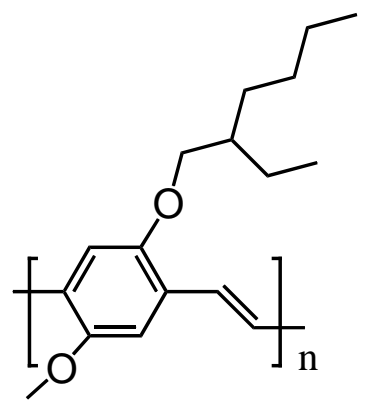


C. TON-THAT et al.

Figure 2

(a)

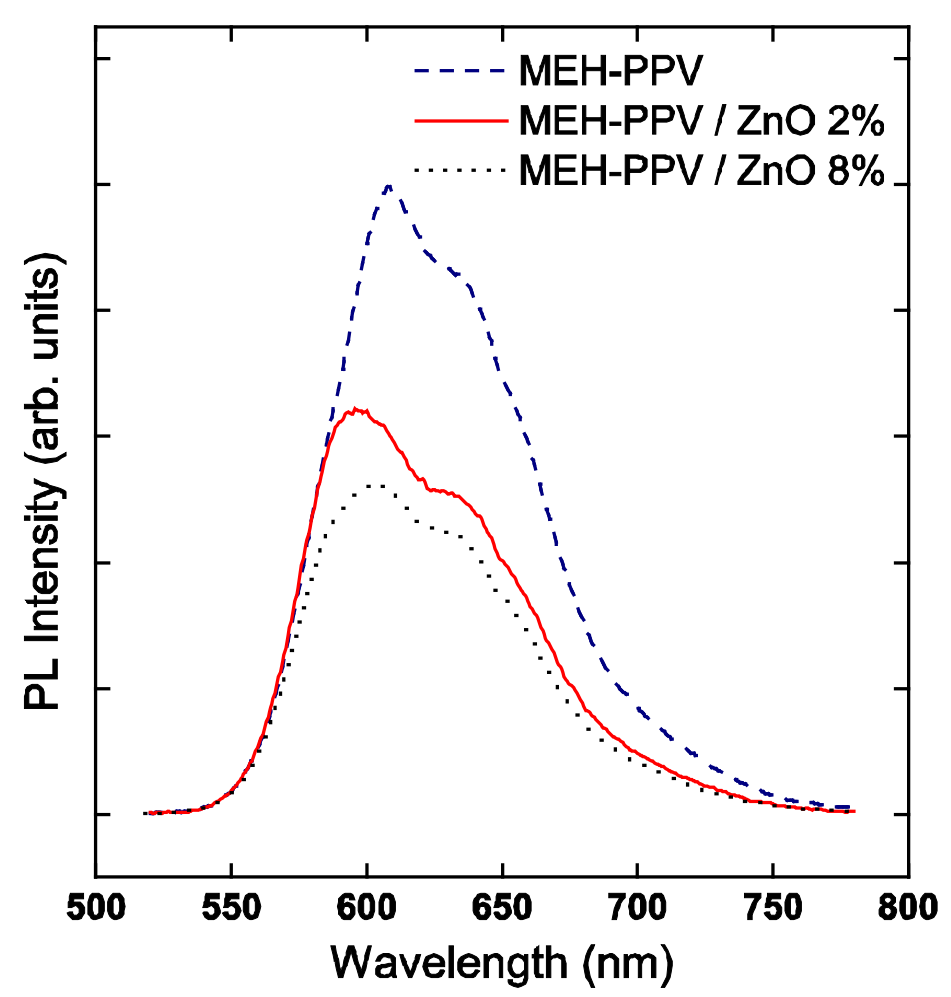

(b)

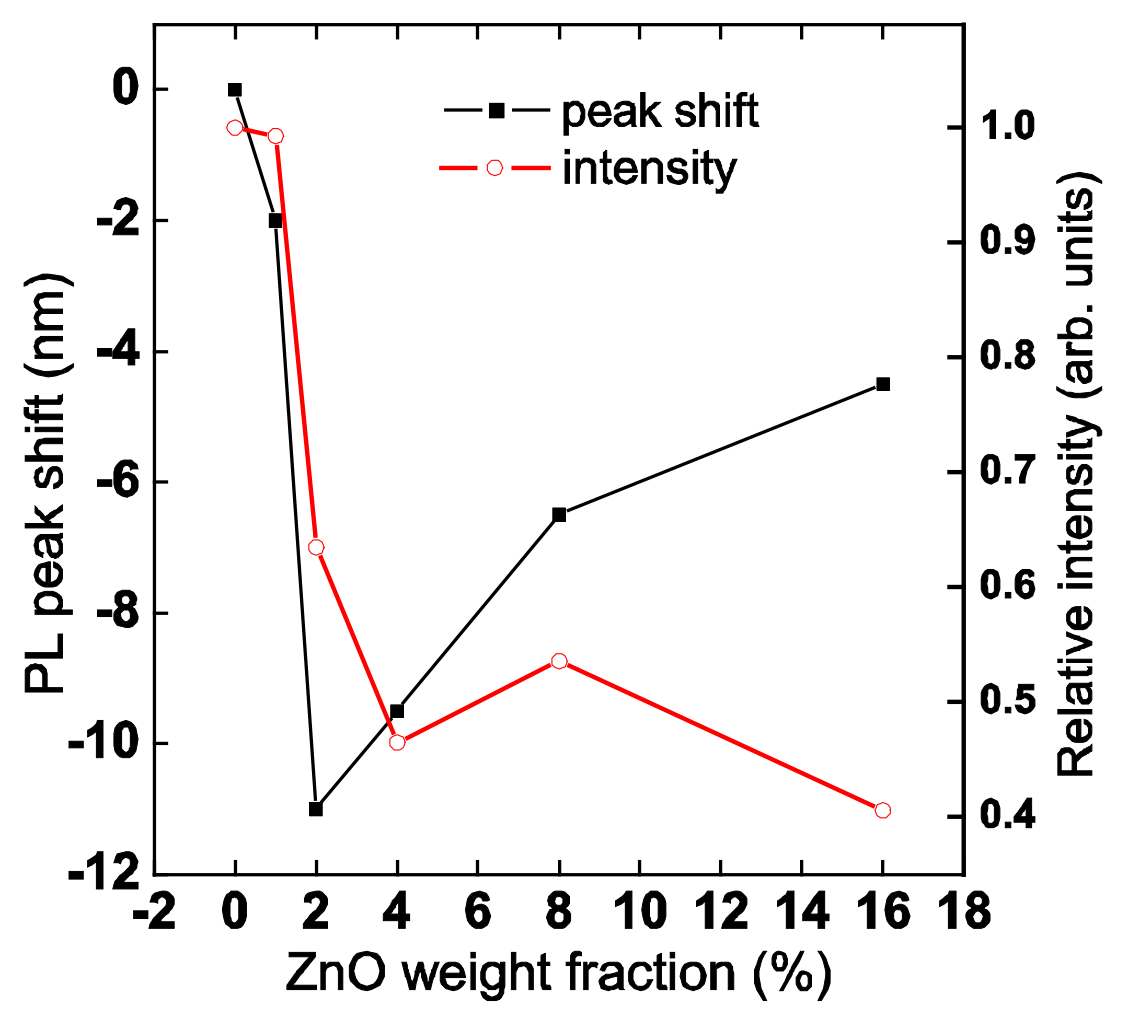


C. TON-THAT et al.

Figure 3

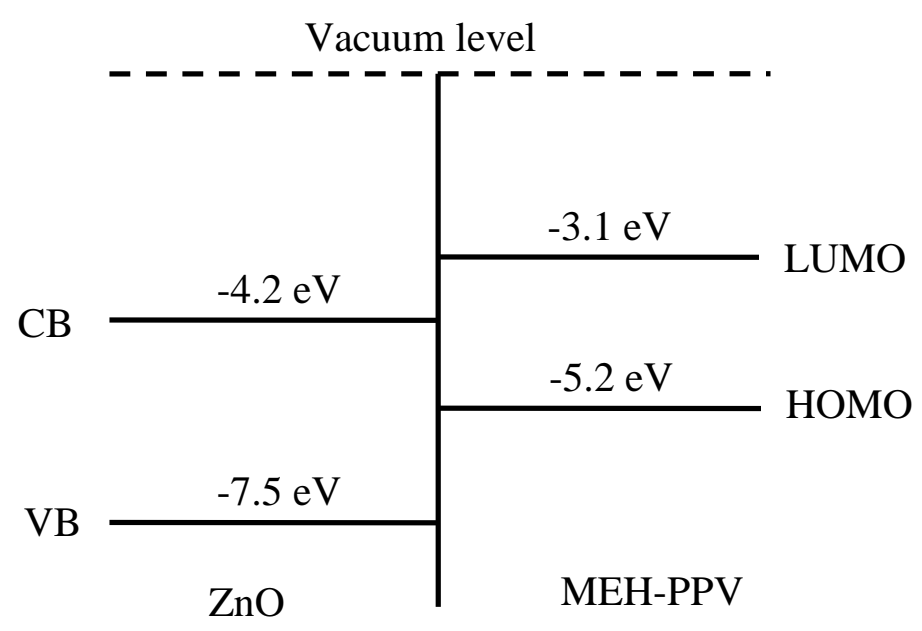


C. TON-THAT et al.

Figure 4

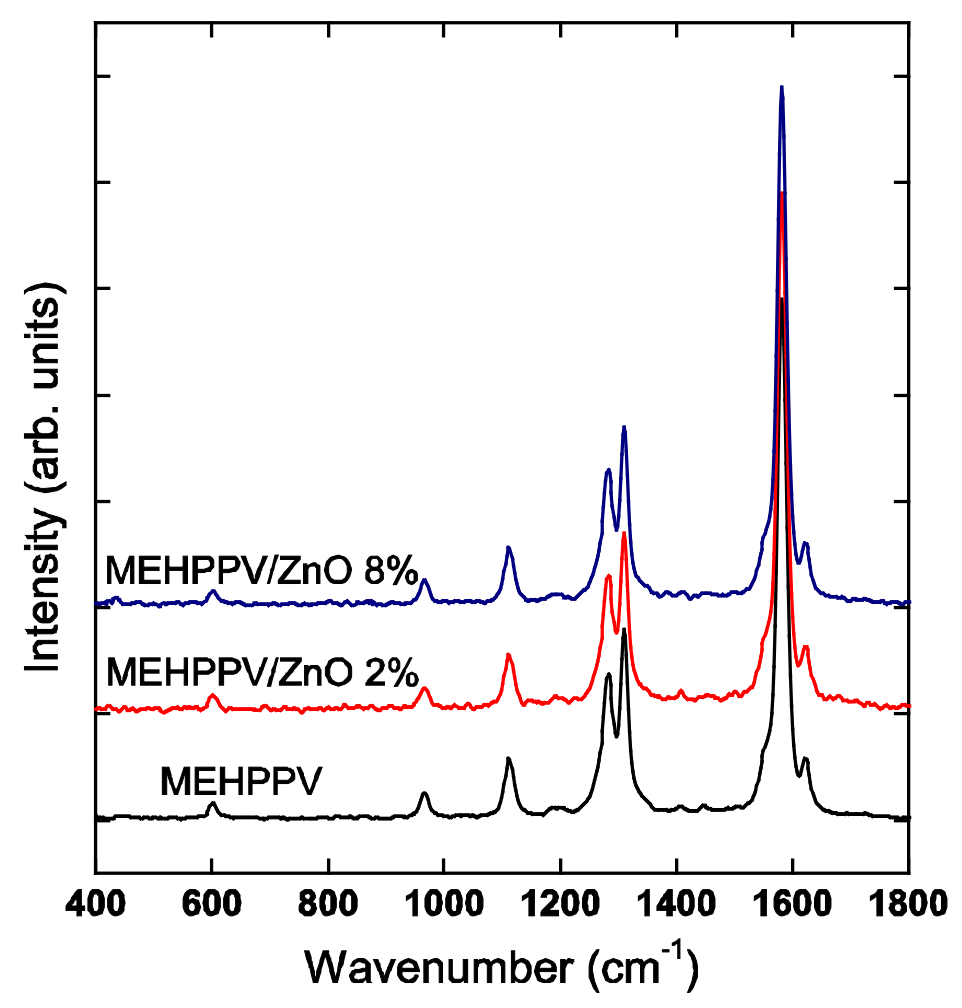

